Décadrages Décadrages

cınéma, à travers champs Cinéma, à travers champs

3 | 2004

Hitchcock côté cour

\title{
Mais im Bundeshuus, un documentaire au service du récit
}

Alain Boillat et Laurent Guido

\section{(2) OpenEdition}

Journals

Édition électronique

URL : http://journals.openedition.org/decadrages/560

DOI : $10.4000 /$ decadrages. 560

ISSN : 2297-5977

Éditeur

Association Décadrages

Édition imprimée

Date de publication : 10 avril 2004

Pagination : 86-97

ISBN : 978-2-9700582-0-5

ISSN : 2235-7823

Référence électronique

Alain Boillat et Laurent Guido, « Mais im Bundeshuus, un documentaire au service du récit », Décadrages [En ligne], 3 | 2004, mis en ligne le 29 avril 2013, consulté le 02 mai 2019. URL : http://

journals.openedition.org/decadrages/560 ; DOI : 10.4000/decadrages.560

(B) Décadrages 
Mais im Bundeshuus, un documentaire au

\section{service du récit}

\section{par Alain Boillat et Laurent Guido}

1 L'expression "transformer un sujet a priori chiant à mourir en un formidable suspense" du Matin (17.9.03) connaît des variantes plus subtiles mais tout aussi révélatrices de cette rhétorique de la "bonne surprise" dans Le Nouvelliste (14.09.03), Femina (14.09.03), L'Hebdo (18.09.03), la Neue Luzerner Zeitung (18.09.03), le Züri Tipp (18.09.03), la Sonntagszeitung (14.9.03), l'Anzeiger Luzern (19.09.03), La Côte (26.9.03) et Filmbulletin (sept. 03).

Ce type de discours vise à mettre en avant l'inventivité du cinéaste. Une phrase comme "une démarche originale qui réinvente le documentaire" (L'Educateur, 29.08.03) ne témoigne pas seulement d'une méconnaissance de l'histoire du cinéma, mais aussi d'une volonté de souligner combien ce documentaire-là représente un cas à part.

2 On remarque aussi que la presse témoigne fréquemment d'un intérêt pour le cinéaste luimême qui occupe une place importante dans les illustrations (Construire lui consacre par exemple sa couverture le 9.09.03) alors qu'il n'apparaît qu'en voix off dans son film. Fait assez rare pour quelqu'un œuvrant dans le cinéma documentaire, Jean-Stéphane Bron est l'objet d'un important processus d'auteurisation, phénomène qui est plus marqué dans le champ de la fiction.

3 Par exemple dans le Tessiner Zeitung (9.08.03), le Corriere del Ticino (9.08.03), Der Bund (9.08.03), le Berner Zeitung (11.08.03), Der Landbote (18.09.03), L'événement syndical (10.09.03), le Tages Anzeiger (17.09.03) ou l'Anzeiger Luzern (19.09.03). On trouve également, de manière dominante, la forme composée "thriller politique", dans la Basellandschaftlische Zeitung (8.08.03), l'AGEFI (8.08.03), le Corriere del Ticino (11.08.03), L'EXpress (11.08.03), Le Matin (4.09.03, 14.09.03
Le succès tant public que critique du dernier long métrage de Jean-Stéphane Bron (voir l'entretien dans ce numéro) incite à s'interroger sur ses particularités formelles et leurs implications esthétiques et idéologiques. Pour étayer l'accueil très favorable qu'elle a réservé au film, la presse helvétique a essentiellement mis l'accent sur son caractère divertissant, afin de contrer implicitement (tout en réaffirmant explicitement) les préjugés liés à une vision restrictive du documentaire comme pratique didactique ou pédagogique (et donc ennuyeuse). En effet, la plupart des articles commencent par affirmer combien le cinéaste a su rendre attractif ce sujet a priori des plus rébarbatifs que sont les discussions dans les coulisses du Palais Fédéral et la gestation d'une loi1. Les termes utilisés, parfois directement inspirés du dossier de presse, sont révélateurs de l'intérêt porté à la structure du film ${ }^{2}$. Mais im Bundeshuus est fréquemment qualifié de «thriller»3 - référence à un genre associé au régime fictionnel - et donc loué pour son "suspense " $\mathbf{4}$ ou, en Suisse alémanique, pour son côté "spannend» ("captivant", "Spannung» signifiant "tension»)5. Lorsqu'on ne recourt pas à une terminologie issue du domaine théâtral ("dramatisation", "dramaturgie», etc.), on va jusqu'à relever la parenté de l'organisation du film avec la fiction 6 . La récurrence de ces critères d'évaluation, conséquence d'un véritable consensus autour d'une notion ("thriller») jamais remise en question, est symptomatique d'une volonté de légitimer la présence d'un film documentaire dans les salles, celles-ci étant en général dominées par une écrasante majorité de films de fiction. En outre, l'aperçu de cette réception critique met à jour d'importants flottements propres aux discours sur le documentaire dans ses liens à la question du récit. Mais im Bundeshuus constitue en ce sens un objet tout à fait approprié à une réflexion sur le rôle de la narration : transforme-t-elle le documentaire en fiction? Les principes narratifs sont-ils nécessairement différents lorsqu'il s'agit d'un film non-fictionnel ? Nous répondrons par la 
négative à ces deux questions à travers l'examen de certains mécanismes narratifs du film de Jean-Stéphane Bron.

Un distinguo initial s'impose qui trouvera confirmation dans notre analyse de Mais im Bundeshuus : les notions «fiction" et "narration" ne sont nullement équivalentes, ni incompatibles. En effet, la fictionalité touche au degré de réalité du représenté, c'est-à-dire au rapport qu'entretient le monde du film avec son référent, alors que le récit a trait au mode d'organisation des différentes parties dont le film est constitué7. Ces deux concepts ne se situent donc pas au même niveau: on peut très bien, du moins dans la théorie (le récit étant le mode dominant), offrir au public un monde créé de toutes pièces sans que l'articulation filmique n'obéisse à des normes narratives (par exemple dans une configuration descriptive ou argumentative); par ailleurs, il est possible d'obéir à une organisation narrative dans l'intention de gérer une matière filmée "réelle» (c'est la dispositio de la rhétorique classique). Dans le cas du film de Bron, il est évident que la quantité d'images tournées (près d'une centaine d'heures) nécessitait une gestion attentive de la temporalité. On peut bien sûr s'interroger sur l'existence de cette "réalité» qui aurait préexisté au film: il est évident que la "neutralité» absolue n'existe pas, même en Suisse, la seule présence du filmeur influençant inévitablement les personnes filmées. D’ailleurs, Bron ne s'est pas caché d'avoir fait répéter de nombreuses fois les politiciens, faisant plusieurs prises comme sur le tournage d'un film de fiction. Cette pratique ne change néanmoins rien au fait que les personnes interviewées et l'objet dont elles parlent (les séances de la commission parlementaire et la préparation de la loi sur le génie génétique, puis sa votation) aient une existence effective (attestée même au niveau juridique), cet ancrage réel étant attesté tant au niveau spatial (le Parlement Fédéral à Berne) que temporel (d'automne 2001 à juin 2002). Néanmoins, tout spectateur habitué aux intrigues des films de fiction aura tendance à rapprocher Mais im Bundeshuus de ces derniers. C'est bien cet effet intertextuel qui esquisse une parenté entre ce film documentaire et la fiction, non la fictionalité propre au film même. Ce détour par les références au régime fictionnel nous permet alors de lancer un pont entre narration et fiction, notamment en nous interrogeant sur la façon dont Jean-Stéphane Bron recourt aux «ficelles» du cinéma de fiction dominant pour guider son spectateur à travers les péripéties politiques qu'il narre. Le modèle de récit canonique auquel il se réfère, s'il ne fictionalise pas le contenu du film, présente des points communs évidents avec ce que l'on trouve habituellement dans les produits les plus calibrés. Voire trop évidents. En effet, la référence et 17.09.03), Filmbulletin (sept. 03), la Neue Luzerner Zeitung (11.08.03), le Zürcher Oberländer (19.09.03) et La Côte (26.9.03). Signe de l'importance de ce terme et de sa fonction d'accroche au lecteur: parmi les articles cités, "thriller" apparaît neuf fois dans le titre ou le chapeau. D'autres expressions faisant également allusion au cinéma hollywoodien ou de divertissement sont parfois utilisées, comme celle de "western fédéral " brandie dans le titre du Journal du Jura (19.09.03), unique modification d'importance apportée à un article déjà paru dans une version plus longue du Nouvelliste (14.09.03).

4 Ainsi le film est-il considéré comme "rythmé, jouant sur des effets de suspense " (La Liberté, 15.09.03) ou "non dénué d'un certain suspense" (Femina, 14.09.03). II "se regarde comme un film à suspense" (24 Heures, 17.09.03); "résultat: un authentique suspense politique" (Le Temps, 17.09.03).

5 L'adjectif "spannend" est employé de façon quasi systématique dans la presse germanophone (une douzaine d'occurrences dans les articles mentionnés ci-dessus).

6 Dans l'entretien réalisé dans Le Matin (14.09.03), trois des quatre questions posées au cinéaste comportent le terme "fiction". Norbert Creutz place cette interrogation dans le titre même de son article (Le Temps, 17.09.03): "La politique fédérale sur grand écran: documentaire ou fiction?". La comparaison menée par André Grieder dans le $n^{\circ} 37$ de Facts entre le film de Bron et le hit du moment en Suisse alémanique, la comédie Achtung, fertig, Charlie (Mike Eschmann, 2004), au profit du documentaire jugé plus drôle, s'inscrit dans une polémique visant à favoriser la production documentaire censée être plus en adéquation avec une certaine spécificité nationale. Nous n'entrerons pas dans ce débat, même s'il est clair que le travail sur le récit permet à Bron de toucher un plus large public sans avoir à être produit par une filiale de Disney.

7 Pour un approfondissement théorique de ces notions, voir Alain Boillat, La fiction au cinéma, L'Harmattan, Paris, 2001. 
marquée à des modes de narration conventionnels pointe la dimension réflexive du film. Les rouages qui y sont exhibés sont bien plus ceux du récit traditionnel que ceux de la politique.

\section{L'entrée du spectateur au Parlement}

8 L'article de Roger Odin porte sur Partie de campagne de Renoir. Cf. La théorie du film, Jacques Aumont et Jean-Louis Leutrat (éd.), Albatros, Paris, 1980.

9 Le recours à la voix off se rapproche ici de son usage "auctorial" chez des cinéastes comme Guitry, Welles ou Truffaut qui prêtent leur propre voix à leurs narrateurs. Ainsi se dessine une zone de recoupement entre des fictions qui laissent percer un peu du réel et un documentaire qui lorgne du côté d'un procédé communément utilisé dans des films fictionnels.

10 Pour l'analyse d'un début de film qui présente une certaine parenté avec celui-ci (l'avancée à travers les portails gothiques de La neuvième porte de Polanski), voir La fiction au cinéma, op. cit., p. 65-69.

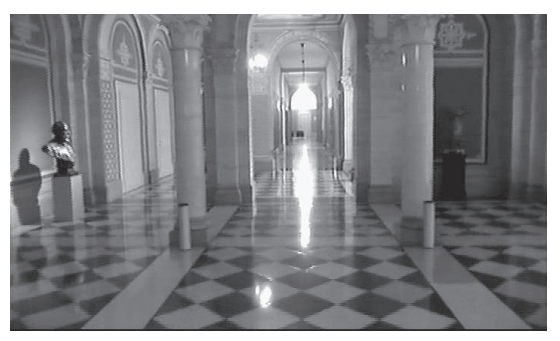

UNE ENTRÉE FLUIDE DANS LE FILM, EN TRAVELLING AVANT
Dans un article intitulé "L'entrée du spectateur dans la fiction»8, Roger Odin s'interrogeait d'un point de vue «sémio-pragmatique» sur les modalités régissant l'immersion progressive du spectateur dans le monde du film. On ne peut toutefois pas dire, comme le suggère Odin dans son article, que les stratégies mises en œuvre soient propres à la seule fiction: le prologue de Mais im Bundeshuus montre combien un documentaire est susceptible de travailler avec précision ce moment-clé où certains indices déterminent le rapport du spectateur au film.

Mais im Bundeshuus ne débute pas sur des cartons de générique qui, nettement séparés des images qui lui succèdent, différeraient l'entrée du spectateur en lui fournissant l'ensemble des informations nécessaires à l'authentification de la réalité filmée. Bron préfère transmettre ces données via une voix off chuchotante qui maintient une certaine proximité au spectateur, tout en constituant un des procédés identifiés comme appartenant à la narration classique ${ }^{9}$. L'absence d'interruptions du flux visuel par des mentions écrites pourtant fréquentes dans les documentaires contribue à mettre l'accent sur l'une des priorités de l'ensemble du film : la linéarité.

Après deux brèves mentions sur fond noir, le film s'ouvre en fade in sur un travelling avant qui nous fait parcourir un couloir du Palais Fédéral. Le passage de l'obscurité à la lumière ainsi que le mouvement vers l'avant tendent à reproduire dans le film même les principes de l'immersion spectatorielle. La construction perspectiviste forte du hall, soulignée par deux colonnades entre lesquelles file la caméra ${ }^{\mathbf{1 0}}$, marque l'importance de cette entrée dans le film. Une instance nous guide ostensiblement, nous tient par la main pour nous présenter les lieux. L'intention est claire : il ne s'agit pas tant d'une déambulation à la Marienbad - ce travelling n'est d'ailleurs pas spécialement long (environ douze secondes) - que d'un plan d'ouverture endossant une fonction précise «d'introduction» du spectateur. Comme par la suite, c'est l'efficacité qui prime: Bron ne s'attardera pas sur les moments «creux» de la Salle des Pas Perdus, mais il mettra l'accent sur des informations qui étoffent la psychologie de ses «personnages» ou influencent le déroulement de l'intrigue.

$\mathrm{Au}$ travelling succède une série de plans fixes qui poursuivent la description de cet espace de la tradition (statues, drapeau suisse, etc.). 
Les lieux sont encore vides, mais le tintement de clochettes qui se fait entendre articule par sa simplicité et son rythme isochrone l'idée d'origine (la naissance du film) avec une certaine temporalité. L'effet de suspension ainsi provoqué crée une sorte d'appel à voir ces différents lieux emblématiques occupés par des individus que la voix off, après avoir ancré les images dans leur contexte spatio-temporel (le 14 octobre 2001 au Palais Fédéral), convoque en parlant des "héros de ce film». Les vues plongeantes qui soulignent l'aspect monumental de l'édifice font alors place au point de vue des futurs occupants. Le terme "héros» est révélateur de la démarche du film: pour assurer une narration classique, il faut mettre en place une individualisation des personnages auxquels le spectateur pourra s'identifier. Le passage du collectif au particulier s'effectue dans ces premières phrases : il est d'abord question d'une commission de 25 parlementaires, puis des «héros» qui ont été choisis parmi ceux-ci pour le film. La salle des commissions, qui restera par la suite toujours hors-champ, subit grâce au cadrage et au montage une importante fragmentation (la gauche puis la droite de la table, renvoyant au clivage politique qui sera l'un des moteurs du récit, puis des casiers avec les trois langues nationales renvoyant aux différences linguistiques) qui culmine dans deux plans où la netteté passe d'un objet à un autre: dans le premier, la mise au point est faite sur le siège, le micro apparaissant flou; dans le second, c'est exactement l'inverse. Or, c'est justement à la collure même de cette seconde image que le mot «héros» est prononcé, au moment précis où l'on passe du siège qui incarne le versant institutionnel du travail des politiciens (la fonction) au microphone, lieu de la parole individuelle et de l'engagement personnel, ce qui annonce le fait que les personnages ne se construiront pas en actes, mais à travers leur parole. Ce déplacement situe les enjeux du film relativement aux convictions d'individus, au détriment de la description d'une mécanique parlementaire désincarnée, d'une analyse de l'institution comme on en trouve par exemple chez Frederick Wiseman.

\section{Chacun sa chance : la multiplication des points de vue}

Cette ouverture fait office de pré-générique puisque, après un fade out qui assure la clôture de cette partie (un signe de démarcation qui permet de clarifier les articulations), apparaît la mention «MAIS IM BUNDESHUUS (LE GENIE HELVETIQUE), ein film von jean-stéphane bron»11. Après ce titre arrive la vue attendue pour le début du film (notamment parce qu'elle est utilisée pour l'affiche) : le Palais Fédéral filmé depuis l'extérieur. Dès le début du film, le spectateur était plongé au cœur du lieu: désormais, il s'agit d'y entrer
11 Notons le privilège accordé dans ce titre au dialecte suisse-allemand: si les deux expressions constituent un jeu de mots, la version allemande est située au-dessus de la version française rejetée entre parenthèses; par ailleurs, les caractères sont plus grands et larges dans "Mais im Bundeshuus", et ont également droit à une couleur vive, le jaune, et à un jeu graphique sur les "u " (désordre renvoyant à l'expression "Mais im...." que l'on pourrait traduire par "faire du foin au..."), alors que le titre français tracé en blanc passe inaperçu ("ein film von" n'étant pas traduit). Ces choix graphiques nous semblent révélateurs de l'intention du cinéaste romand d'avoir un impact à un niveau national. 
12 Voir David Bordwell, Narration in the Fiction Film, Routledge, University of Wisconsin, 1985, p. 56-57.

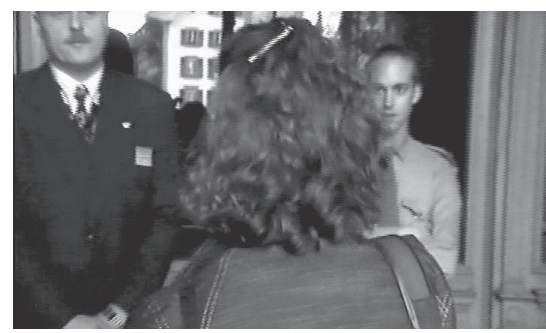

MAYA GRAF AUX PORTES DU PALAIS FÉdÉraL véritablement, non plus en adoptant le point de vue déshumanisé de la caméra, mais en accompagnant un personnage, Maya Graf. Son nom, d'abord prononcé par un agent de la sécurité, est ensuite répété par la voix off assorti du prénom (première marque d'individualisation), pour apparaître enfin en surimpression avec la mention du parti écologiste qu'elle représente: ces trois occurrences, diverses de par leur mode d'énonciation, participent du principe de redondance caractéristique du récit classique $\mathbf{1 2}$ qui est garant d'une lisibilité optimale. Ce n'est pas un hasard si le film commence (et se termine) sur Maya: comme le dit la voix off, elle est "fraîchement élue", et constitue donc un intermédiaire approprié pour introduire le spectateur au Parlement. Comme lui, elle manque de repères et demande son chemin à un garde. Le choix de Maya comme protagoniste initiale arrime également le point de vue politique du film. Toutefois, la sympathie pour la gauche n'infléchit pas chez Bron la représentation de la palette des représentants politiques: le cinéaste opère une juxtaposition des points de vue qui offre à chacun sa chance. Sur ce point, qui est peut-être le principe structurant fondamental de la première partie de Mais im Bundeshuus, Bron renoue avec la méthode dialectique qu'il applique dans ses films antérieurs: dans Connu de nos services, il juxtapose par exemple un récit où la compagne de Claude Muret détaille le moment où celui-ci lui a annoncé son départ pour le Viêt-Nam et l'extrait d'un entretien où Muret luimême dit ne plus se souvenir de rien; dans La bonne conduite, où le procédé fait véritablement système, ce sont les perceptions différenciées qu'ont les moniteurs d'auto-école d'une part, leurs élèves d'autre part. Cet enchevêtrement de points de vue ne prétérite personne: comme le radical proche des lobbies pharmaceutiques Randegger dans Mais im Bundeshuus, le chef du corps de police Hartmann dans Connu de nos services et le moniteur affectionnant les allusions racistes de La Bonne conduite ont droit à la parole et à un approfondissement qui permet de nuancer leur position. Ce refus de tout manichéisme (qui confine dans Mais im Bundeshuus à une certaine attirance pour le "camp adverse") nous semble tout entier contenu dans deux plans de La bonne conduite où, après un passage où l'élève d'origine hindoue souffrant des pointes xénophobes de son moniteur, Cherix, s'interroge sur ce dernier tout en s'en rapprochant ("on se dit mon gaillard, toi tu caches quelque chose dont tu n'as pas envie de parler, comme moi»), on nous montre Cherix seul dans un pâturage, contemplant le ciel qui se couvre. Le plan des nuages introduit un raccord-regard qui exprime cette volonté de rester solidaire des personnages, fussent-ils abominables. Cette respiration silencieuse constitue un espace de liberté que le cinéaste réserve à ceux 
qu'il filme, même lorsqu'il s'agit d'exprimer, comme ici, l'indicible parce que refoulé. La démarche de (ré)conciliation engagée par Bron peut s'interpréter comme une volonté de prendre en compte cette part de l'identité nationale habituellement rejetée dans le champ culturel auquel appartient le cinéaste. Dans Mais im Bundeshuus, on trouve ainsi deux visages emblématiques d'un certain conservatisme typiquement helvétique: la Suisse profonde (Kunz) et les milieux économiques zurichois (Randegger).

Cette esthétique kaléidoscopique permet alors de comprendre l'intérêt de Bron pour la Gen-Lex (et son désintérêt pour les détails de sa genèse): créer une loi, c'est avant tout trouver un consensus, fruit d'une compréhension mutuelle. Le début du film renvoie à cette notion en faisant converger dans le Parlement les différents protagonistes qui arrivent successivement (dans l'ordre, après Graf: Kunz, Randegger, Chappuis, Neyrinck), une technique qui permet par ailleurs de préciser de manière extrêmement claire les liens spatio-temporels unissant les cinq "pistes": chaque nouveau parlementaire est situé par rapport au premier repère que représente Maya mangeant une pomme, seule, dans le corridor (une situation qui souligne l'extériorité de la néophyte). En effet, elle réapparaît pour chaque piste à l'arrière-plan ou en insert, dans le respect le plus scrupuleux des règles d'expression de la simultanéité selon la "grammaire» hollywoodienne. Par ailleurs, "l'harmonie classique» est soutenue par le principe de symétrie: pour chacun des protagonistes, le texte évoque leur situation privée en accompagnant l'information d'une sorte de "photographie» filmée. Posant immobiles, Maya Graf et Joseph Kunz sont filmés en famille devant leur ferme, ce qui donne à ces images figées une valeur archaïsante en adéquation avec le propos (l'origine terrienne). Ce procédé est déjà à l'œuvre dans trois documentaires ayant trait à la paysannerie suisse : Der grüne Berg (Fredi Murer, 1990) et deux films de Stéphane Goël, A l'Ouest du Pécos (1993) et Campagne perdue (1997)13. Le rapprochement permet aussi l'opposition: la disposition désordonnée des figurants de la «smala» Graf, dont l'apparence des petits et grands révèle un milieu simple, contraste avec l'aspect solennel de la «dynastie» Kunz campée par les parents et les fils devant une série de photos suspendues au mur telles des trophées, à côté d'une fontaine ornée d'une date. Le texte met d'ailleurs en évidence la dimension plus commémorative de cette image en disant de Joseph Kunz que "son exploitation est aux mains de ses fils, mais il règne sur ce domaine où vit et meurt la famille Kunz depuis le XVI ${ }^{\mathrm{e}}$ siècle». Cette phrase témoigne par son emphase d'une attirance évidente de Bron pour
13 Sur les liens esthétiques et narratifs qui unissent certains documentaires lausannois des années 1990, et sur leur questionnement commun de l'histoire du cinéma suisse, voir Laurent Guido, "Video als Blickpunkt", Cinema, $n^{\circ} 46$, p. 55-66 (Heimspiele. Film in der Schweiz seit 1984). 

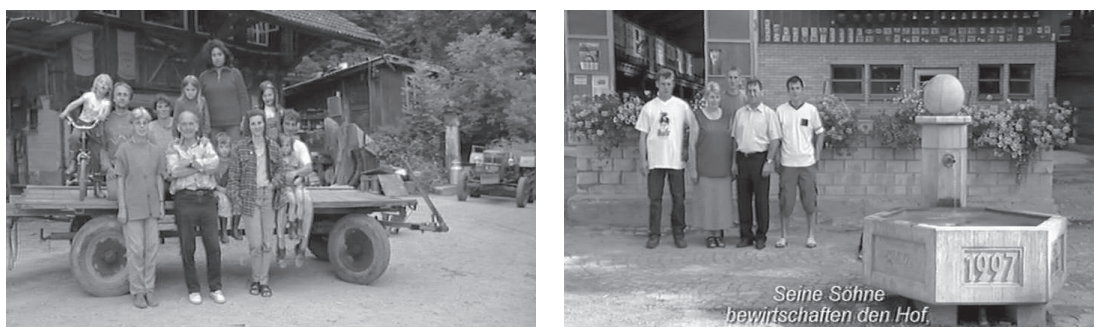

DEUX IMAGES CONTRASTÉES DE LA FAMILLE PAYSANNE: LA "SMALA" GRAF ET LA «DYNASTIE" KUNZ

l'enracinement séculaire, mais elle s'inscrit également dans une optique narrative, puisque l'un des retournements de situation ultérieurs passera par l'alliance "paysanne» entre l'écologiste et le représentant de la droite traditionaliste. Ce rapprochement sera d'ailleurs marqué par une directive explicite du cinéaste qui témoigne de l'importance qu'il accorde à la communauté d'intérêts propre à la paysannerie, par-delà les partis : après un plan où l'on voit Maya souffler quelques mots à l'oreille de son cheval, c'est la voix de Bron qui chuchote pour informer Kunz que le cheval de celle-ci est malade. Ainsi, même ce qui peut apparaître a priori comme des digressions personnelles ou des échos à portée symbolique s'avère être, in fine, au service du récit.

Lors de leur arrivée au Parlement, tous les acteurs de ce puzzle politique ne sont cependant pas montrés de la même manière. Celui que l'on nomme le "Professeur Neyrinck» arrive avec un certain retard, annonciateur de la position de commentateur distancié qu'il endossera dans l'ensemble du film, en bon représentant de l'homme de savoir et du parti centriste. Mais c'est surtout Johannes Randegger qui est mis en évidence comme le bad guy au service de Novartis-Syngenta: il est le seul à arriver encadré de son team, alors que retentit une mélodie à la guitare électrique qui rappelle celles écrites par Ennio Morricone pour des westerns spaghetti. Contrairement à Maya dont nous suivions la marche

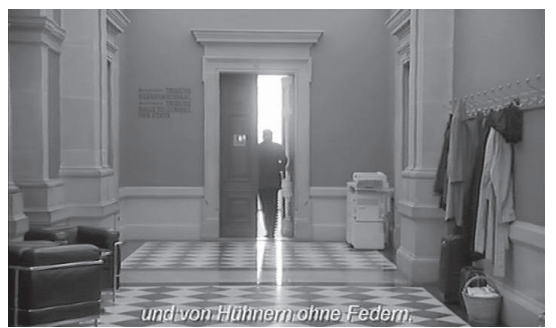

NEYRINCK LE RETARDATAIRE ACCÈDE À LA SALLE DE LA COMMISSION, LE HORS-CHAMP DU FILM

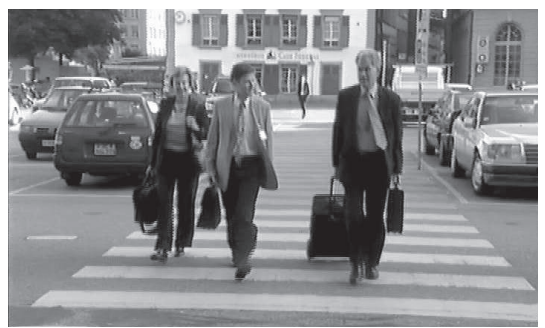


de dos, Randegger, filmé à distance et frontalement, s'avance contre nous, en prédiction du duel qui l'opposera à cette dernière. Les inserts illustrant son milieu (notamment un intérieur design aux couleurs froides) le présentent comme appartenant à une élite, comme l'homme de l'industrie (on nous précise que, bien qu'indépendant, il a conservé un bureau chez le géant pharmaceutique). Incarnation de l'Opposant nécessaire à tout récit, le radical - personnalisé jusqu'à la quasi-caricature par ses tics de "carnassier» (chewing-gum en bouche) et sa démarche assurée - connaîtra néanmoins, plus tard dans le film, une sorte de réhabilitation qui sied à la démarche de Bron. Il s'agit d'un entretien dans lequel le parlementaire dévoile son passé et explique son rapport à la défaite. En abordant des éléments intimes, le bad guy se teinte d'humanité, ce qui le rend plus sympathique aux yeux du public. Dans ce segment consacré à Randegger (chacun des quatre parlementaires principaux dominant l'une des parties du film) qui commence par un plan nous le montrant seul, de profil, au volant de sa voiture, il est intéressant de noter que le moment d'humanisation correspond à un renversement : le radical vient de perdre une bataille. Le personnage gagne donc en intérêt au moment où il faiblit, son assurance faisant jusque-là obstacle à l'identification du spectateur. Ce sont désormais les partis de gauche qui sont montrés comme manipulateurs, et notamment Maya Graf qui de séductrice ingénue devient une véritable lady Macbeth. L'inversion provisoire, manifeste dans le plan qui suit l'entretien de Randegger où on le suit de dos comme Maya au début du film, participe des procédés utilisés par le cinéaste pour découper son film en grandes phases narratives. A la fin, le coup de théâtre des votations prendra le relais de ces moments où des conflits se nouent: la réalité elle-même fera office de potentiel dramatique.

Comme on l'a montré dans les quelques passages traités, la manière d'introduire puis de filmer les individus tient compte des développements ultérieurs, s'inscrivant fortement dans une logique de type narratif. L'assemblage pirandellien d'opinions divergentes relève à la fois d'un travail ludique sur les possibilités de la focalisation et d'une éthique humaniste. Le spectateur a, lui, une vision globale des différentes stratégies et opinions, si bien qu'il peut jouir, comme dans la plupart des récits de fiction (le documentaire étant plutôt communément lié à l'aléa), d'une situation privilégiée d'omniscience particulièrement manifeste lorsque Kunz termine sa discussion, restituée au spectateur, en disant «mais ils n'en savent rien encore, c'est mon atout».

Le respect altruiste conduit à une forme de consensus (le "génie helvétique", dira-t-on), puisque le réalisateur ne donne jamais 


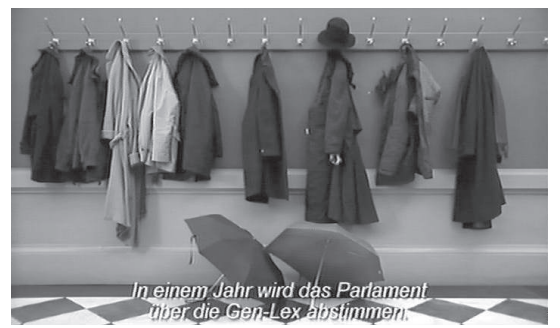

AU LIEU DES DÉBATS, LA GARDE-ROBE explicitement son avis à propos du génie génétique ou de la position d'un parti. L'abdication de tout engagement a d'ailleurs probablement été pour Jean-Stéphane Bron une condition sine qua non pour gagner la confiance des politiciens et obtenir leurs confessions, aspect essentiel de son travail qui vise à faire passer le politique du côté de l'individuel. L'adoption d'un récit canonique, avec ses personnages bien définis et sa progression linéaire, conduit à une réduction du politique, conçu sans véritablement entrer dans ses enjeux les plus larges qui en constituent les rouages. Assurément, Mais im Bundeshuus est plus proche d'un film de Capra que du Cuirassé Potemkine.

\section{La socialiste marginalisée}

L’opposition Maya Graf / Johannes Randegger, deux personnages habiles à l'auto-mise en scène, détermine un axe à l'aune duquel sont jugées les prises de position de Kunz et de Neyrinck susceptibles de faire pencher la balance. Sur les cinq protagonistes présentés au départ, on constate donc que la représentante socialiste, Liliane Chappuis, se tient passablement en retrait, menaçant d'autant plus l'équilibre de la représentation des fractions qu'elle appartient au parti constituant alors la plus grande force politique de Suisse. Probablement trop réservée et insuffisamment typée pour camper un personnage pouvant lutter à armes égales avec les quatre "héros", la socialiste se cantonne principalement, dans les entretiens (plutôt brefs) qui lui sont accordés, à commenter la parole des autres. Ce procédé est certes inévitable dans la mesure où le spectateur n'a pas accès à la salle de conférence, mais les autres protagonistes affirment beaucoup plus nettement leur subjectivité dans leur manière d'en rendre compte. Lorsque Randegger nous informe avec enthousiasme de sa stratégie consistant à faire durer la séance pour reporter le vote, un entretien avec Liliane Chappuis est placé en insert avant que ne reprenne l'explication du radical bâlois. Ce montage est révélateur d'une certaine subordination du discours de Chappuis aux fortes têtes.

Le film nous semble donc confronté au problème de la représentation du PS. Preuve en est l'intervention d'autres figures du parti qui ont également droit à une surimpression écrite de leur nom, telle que Rudolph Stramm, Simonetta Sommaruga ou Anita Fetz, comme si le cinéaste prenait conscience de la nécessité de donner plus de poids à cette fraction. Lors des deux discussions simultanées où les socialistes attaquent Kunz, Chappuis n'est pas de la partie ; plus tard, c'est à nouveau Anita Fetz qui menacera le représentant de l'UDC de ne pas le soutenir dans un projet de loi ultérieur s'il n'est pas cette fois-ci de leur côté. Figure plus offensive, Fetz aurait pu endosser de façon plus 

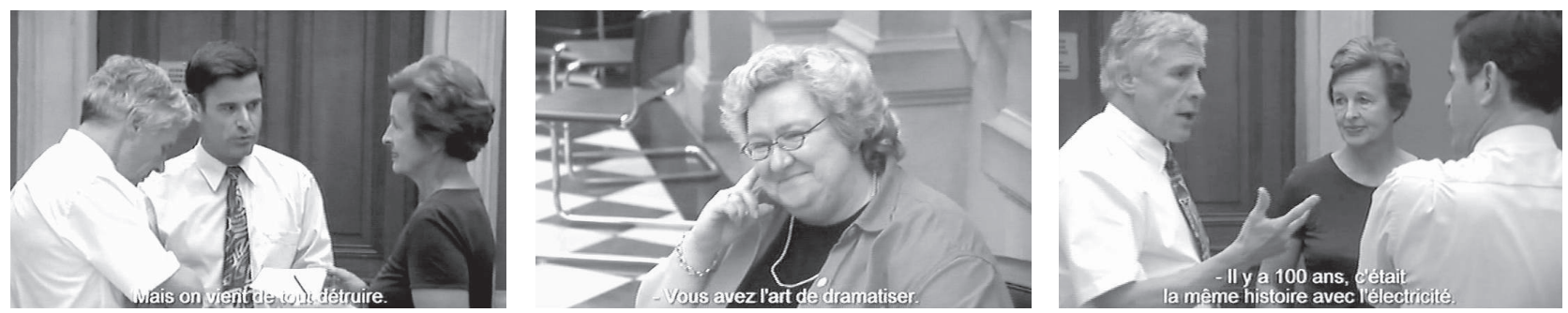

LE REGARD EXTÉRIEUR DE LILIANE CHAPPUIS INSÉRÉ DANS UN DIALOGUE

adéquate le rôle d'un "Randegger de gauche». Liliane Chappuis paraît coupée de toute véritable interaction avec d'autres politiciens. Lorsque deux membres du PS interpellent avec véhémence Kofmel, "l'acolyte» de Randegger, elle n'est introduite dans le débat que de manière artificielle, à la faveur d'un contre-champ muet où on la voit sourire. Un plan de cette discussion à trois a été utilisé pour une image reprise dans le 24 Heures du 9-10 août 2003 qui en côtoie cinq autres. Dans la représentation synthétique des six photographies proposée par ce journal, il faut noter que Chappuis n'apparaît pas, alors que les cinq autres protagonistes sont présents (Randegger apparaissant dans trois d'entre elles). La parlementaire socialiste, qui n'a pas été réélue dans l'intervalle, ne se prête pas au même show que les autres. Pourquoi le choix de Bron s'estil porté sur elle? Peut-être voulait-il une femme et une Romande, afin d'une part de renforcer l'opposition entre la droite virile et la gauche féminine et, d'autre part, de ne pas trop minorer, comme cela aurait été le cas en choisissant Fetz, la partie francophone de ce film produit à Lausanne. Peut-être souhaitait-il également donner une certaine représentation du Parti socialiste dans son ensemble, vu comme le lieu d'une institutionnalisation des idées de gauche, d'une politique "politicienne» pas si éloignée de celle que conduisent leurs adversaires. C’est du moins l'idée que suggère Maya Graf lorsqu'elle fait remarquer l'apparence vestimentaire très similaire de Stramm (PS) et de Randegger (PR): ils ont "presque la même cravate!»

\section{Les modalités de la captation}

Pour dynamiser la succession d'entretiens qui composent l'entièreté du film, Jean-Stéphane Bron varie la façon de nous faire accéder à la réalité dont il rend compte. Il exploite avant tout deux modes, que nous proposerons d'appeler et de décrire comme suit : le "Mode Entretien" (ME) où les politiciens se trouvent face à la caméra et s'adressent à elle; le "Mode Diégétisation» (MD) où le cinéaste surprend (ou feint de surprendre) 


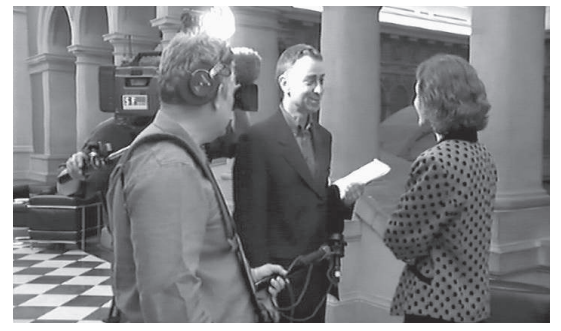

LA MISE À DISTANCE DU REPORTAGE TÉLÉVISUEL une discussion qui n'inclut pas l'instance de filmage, comme si cette discussion se déroulait de manière totalement indépendante du tournage du film. Le premier se situe donc plutôt du côté d'un marquage énonciatif qui renvoie à la présence de l'équipe du film, alors que le second relève plutôt de cet effacement de tout ce qui a trait au tournage qui est caractéristique des fictions. Liliane Chappuis, qui n'est jamais montrée en discussion avec d'autres, ainsi que Jacques Neyrinck, qui garde ses distances tout en entretenant une forte proximité avec le cinéaste, sont très majoritairement filmés en ME. Les trois autres, de même que tous les protagonistes secondaires du film, ont également droit au MD.

En ME, il arrive que la voix du cinéaste se fasse entendre horschamp, notamment lorsqu'il pose des questions destinées à orienter le cours de la discussion dans le sens d'une dramatisation du contenu (la maladie semblant faire office de ressort important: Bron revient sur celle du cheval de Graf et sur celle de Neyrinck). Toutefois, nous ne voyons jamais l'équipe de tournage ou le traducteur, et a fortiori le cinéaste. Bron réduit les marques d'énonciation du film aux regards-caméras et à quelques jump cuts (sautes brusques) qui enfreignent certaines normes "classiques» du montage, mais ces éléments semblent aujourd'hui suffisamment assimilés par le public - composé de téléspectateurs pour qui ces pratiques de rupture sont dominantes - pour pouvoir être conciliés avec une conception linéaire. Lorsqu'on nous montre une équipe, il s'agit de la TV, par exemple SF1 interviewant Maya: ce n'est donc pas le dispositif propre à Mais im Bundeshuus qu'on exhibe, mais celui des autres, ce qui provoque un déplacement du marquage (une mise en abyme) qui contribue, au contraire, à renforcer la transparence, puisqu'on enchâsse en fait le ME dans le MD.

Lorsque les personnages sont seuls, il peut paraître difficile de mettre en place un MD; Bron trouve néanmoins une sorte d'équivalent de l'aparté théâtral en demandant à ses «acteurs " (probablement le sontils plus que jamais dans de tels moments) de parler au téléphone à des proches (que nous n'entendons pas). Cette situation atteint un climax lorsque Maya Graf, pressée de retourner dans la salle de l'assemblée, quitte la cabine téléphonique en laissant pendre le combiné (dans le cas de conversations avec un portable, on reste plus proche du ME). Le MD s'avère donc l'un des instruments de l'immersion du spectateur dans le monde du film, une façon de lui faire ressentir les angoisses et les joies des personnes filmées. Pour qu'ait lieu cette empathie - que le spectateur peut rapprocher de la fiction où les personnages sont spécifiquement créés pour la provoquer -, il faut que la distance due au tournage même s'estompe. Alors, l'identification au personnage bénéficie d'un atout 
supplémentaire sur la fiction: s'adonnant à une lecture documentarisante, le spectateur sait que ces gens existent et ont éprouvé ce qu'ils confessent. Sous le "héros" du film, il y a toujours en même temps la personne réelle. Lorsque ce surgissement du réel se produit dans un film de fiction (par exemple lorsque l'on remarque l'âge d'un acteur, non du personnage qu'il incarne), on aurait tendance à dire qu'il enraie l'absorption du spectateur dans la diégèse, alors qu'ici c'est le contraire. Mais im Bundeshuus tire sa force d'allier des procédés hérités de la fiction à l'ancrage réel d'un documentaire. Une image du film concrétise cette conception d'un monde qui se donne de lui-même: après un plan nocturne, des rideaux s'ouvrent sur une salle baignée de lumière matinale. Non seulement le travail sur la temporalité est affiché, mais nous ne voyons personne tirer les rideaux, ils s'ouvrent d'eux-mêmes. Personne ne semble tirer les ficelles du film comme si la réalité s'offrait à nous sans médiation, alors qu'en même temps les rouages du récit sont sans cesse exhibés, et même presque jusqu'à l'excès. C'est le paradoxe dont se joue Mais im Bundeshuus, bâti comme la plupart des fictions sans quitter le réel. 